\title{
TOXICIDADE AGUDA DO AGROTÓXICO CARBOFURANO AO CLADÓCERO CERIODAPHNIA SILVESTRII DADAY, 1902
}

\author{
Adrislaine da Silva Mansano ${ }^{1}$
}

Raquel Aparecida Moreira²

Odete Rocha ${ }^{3}$

RESUMO: Nos últimos anos o aumento do consumo de agrotóxicos em todo o mundo tem preocupado agências nacionais e internacionais em decorrência dos diversos impactos ambientais e das sérias implicações à saúde humana. Dentre os agrotóxicos, o carbofurano é utilizado amplamente no Brasil em culturas de batata, milho, amendoim, arroz e soja. Como existe o risco deste composto ser carreado por diversos mecanismos até os corpos d'água e causar toxicidade a espécies não-alvo, é necessária a avaliação dos seus efeitos nestes ecossistemas. O objetivo deste trabalho foi avaliar a toxicidade aguda do carbofurano sobre a espécie nativa tropical Ceriodaphnia silvestrii. Para isso, realizaram-se testes de toxicidade aguda, com concentrações nominais deste agrotóxico. Para o dafinídeo $C$. silvestrii, os valores de $\mathrm{CE}_{50} 24 \mathrm{~h}$ e $\mathrm{CE}_{50} 48 \mathrm{~h}$ foram de $1,80 \mu \mathrm{g} \mathrm{L}^{-1} \mathrm{e}$ $0,75 \mu \mathrm{g} \mathrm{L}^{-1}$, respectivamente. Os resultados obtidos mostraram que $C$. silvestrii apresentou elevada sensibilidade ao agrotóxico carbofurano e foi mais sensível do que as espécies de cladóceros de regiões temperadas, de acordo com os dados da literatura. Considerando-se estes resultados e da ampla distribuição de $C$. silvestrii em regiões tropicais e subtropicais, sugere-se o uso desta espécie em avaliações ecotoxicológicas com agrotóxicos em águas doces tropicais.

\footnotetext{
${ }^{1}$ Bióloga. Doutoranda do Programa de Ecologia e Recursos Naturais, Universidade Federal de São Carlos, São Carlos, SP, Brasil. E-mail: laine_mansano@yahoo.com.br

2 Bióloga. Mestranda do Programa de Ecologia e Recursos Naturais, Universidade de São Paulo, São Carlos, SP, Brasil. E-mail: raquel.moreira87@yahoo.com.br.

${ }^{3}$ Bióloga. Docente do Departamento de Ecologia e Biologia Evolutiva, Universidade Federal de São Carlos, São Carlos, SP, Brasil. E-mail: doro@ufscar.br
} 
Palavras-chave: Microcrustáceo. Pesticida. Toxicidade.

\section{INTRODUÇÃo}

A moderna agricultura, que busca constante elevação de produtividade e maximização dos lucros, emprega uma carga expressiva de agroquímicos, dentre os quais se encontram os agrotóxicos, principalmente herbicidas, inseticidas e fungicidas, que podem causar poluição ambiental e desequilíbrio do ecossistema (GRÜTZMACHER et al., 2008).

O uso de agrotóxicos vem aumentando de forma significativa em diversos países, colocando em risco a saúde dos ecossistemas e do homem. De acordo com os dados do Sindicato Nacional da Indústria de Produtos para Defesa Agrícola - SINDAG (2012), as vendas de agrotóxicos no Brasil aumentaram mais de 72\% entre 2006 e 2012 - de 480,1 mil para 826,7 mil toneladas. As vendas destes compostos defensivos movimentaram quase US\$ 8,5 bilhões no Brasil em 2011, tornando-o o segundo maior mercado do mundo, atrás apenas dos Estados Unidos (SINDAG, 2012).

Um dos maiores problemas relacionados com o uso de agrotóxico é que a maior parte aplicada acaba não atingindo os organismos-alvo, sendo carreada pelas águas das chuvas, percolando ou volatilizando, representando uma ameaça aos ecossistemas como um todo, principalmente os aquáticos.

Os agrotóxicos podem classificar-se de vários modos: segundo a praga à qual é destinado, estrutura química do composto utilizado ou a sua toxicidade, grau ou tipo de risco sanitário. De acordo com o tipo de aplicação, os pesticidas podem-se dividir em dois tipos: sistêmicos (circulam no sistema vascular das plantas) e não sistêmicos ou de contato. As classes químicas mais importantes compreendem os compostos organoclorados, organofosforados e os carbamatos. Dentre estes, os carbamatos, como o carbofurano, o aldicarbe e o carbaril, são considerados um dos mais importantes pesticidas, pois são extremamente tóxicos para organismos aquáticos.

No Brasil, o carbofurano é comercialmente encontrado pelo nome de Furadan, Carborano e Ralzer, vendidos em diferentes formulações. O carbofurano (2,3-diidro-2,2dimetil-7-benzofuranil-N-metil carbamato) largamente utilizado em culturas de batatas, 
milho, amendoim, arroz e soja, é um inseticida, acaricida e nematicida do grupo dos carbamatos, cuja estrutura molecular é apresentada na Figura 1.

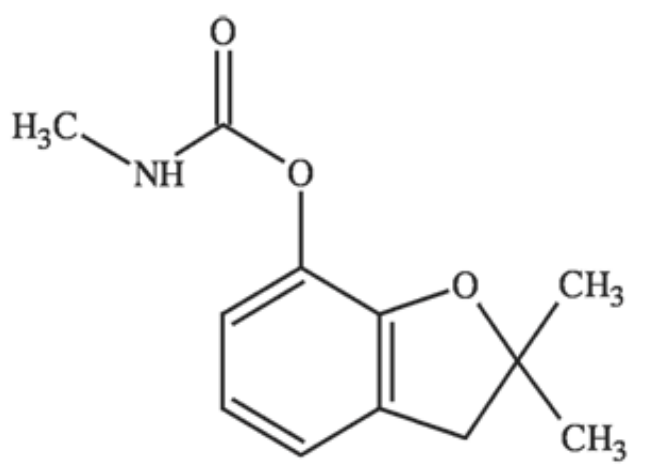

Figura 1: Estrutura química do carbofurano (VALENCIA et al., 2008).

O carbofurano liga-se reversivelmente à enzima acetilcolinesterase, inibindo a ação desta sobre a acetilcolina. Este agrotóxico apresenta curta persistência no ambiente e é classificado como muito tóxico para as abelhas, peixes e outros animais aquáticos e perigoso para animais e aves (COLLECTIVE SPA, 2002). Segundo estudos, devido a sua ampla utilização, o carbofurano foi detectado em águas subterrâneas, superficiais e da chuva, em solos, ar, alimentos e animais (RICHARDS et al., 1987; WAITE et al., 1992; FISHER et al., 1999).

Diante da contaminação dos recursos hídricos por agrotóxicos e outros compostos, a ecotoxicologia surge como uma ferramenta necessária para avaliar a toxicidade destes às comunidades aquáticas. $\mathrm{O}$ uso de cladóceros em testes de toxicidade tem muitas vantagens, como a sua facilidade de manipulação, reprodução partenogenética, ciclo de vida curto e alta sensibilidade a substâncias tóxicas, características que os tornam organismos adequados para bioensaios (TRAYLER e DAVIS, 1996). Os cladóceros planctônicos mais comumente usados em ensaios ecotoxicológicos são Daphnia magna (BAILLIEUL e BLUST, 1999) e Ceriodaphnia dubia (STEWART e KONETSKY, 1998), no entanto, a distribuição geográfica natural destas espécies é restrita a áreas do hemisfério norte (MITCHELL et al., 2004). Assim, neste estudo optou-se por selecionar como organismo-teste a espécie tropical nativa Ceriodaphnia silvestrii que é facilmente encontrada em vários tipos de corpos de água brasileiros. 
Dentro deste contexto, o objetivo deste trabalho foi avaliar a toxicidade aguda do agrotóxico carbofurano ao cladócero nativo Ceriodaphnia silvestrii. Estudos desse tipo podem gerar dados sobre a sensibilidade de espécies nativas e impactos sobre a biota e subsidiar ações futuras que visam proteger os organismos aquáticos neotropicais frente à contaminação por agrotóxicos.

\section{MATERIAL E MÉTODOS}

\subsection{Cultivo e manutenção de Ceriodaphnia silvestrii}

Para o cultivo da $C$. silvestrii foi utilizada água reconstituída preparada de acordo com a ABNT (2005) e com as seguintes características: pH de 7,0 a 7,6, dureza entre 40 e $48 \mathrm{mgCaCO}_{3} \mathrm{~L}^{-1}$ e condutividade elétrica de $160 \mu \mathrm{sm}^{-1}$. Essa espécie de cladócero foi mantida em béqueres de $2 \mathrm{~L}$, com um máximo de 140 indivíduos por recipiente. $\mathrm{O}$ cultivo foi mantido em incubadora com temperatura controlada de $25 \pm 2^{\circ} \mathrm{C}$ e fotoperíodo de $12 \mathrm{~h}$ (claro)/12h(escuro).

Os indivíduos de $C$. silvestrii foram alimentados com suspensão algacéa de Pseudokirchneriella subcapitata, cultivada em meio CHU-12 (MÜLLER, 1972), a concentração de $1 \times 10^{5}$ células $\mathrm{mL}^{-1}$ e alimento composto (levedura e ração de peixe fermentada na proporção de 1:1) numa concentração de $1 \mathrm{~mL} \mathrm{~L}^{-1}$ (ABNT, 2004). A manutenção das culturas foi realizada três vezes por semana, quando eram renovados a água e o alimento. Para avaliar as condições fisiológicas dos organismos-testes, foram realizados mensalmente testes de sensibilidade com a substância de referência cloreto de sódio ( $\mathrm{NaCl})$, segundo as recomendações da ABNT $(2004,2005)$.

\subsection{Testes de toxicidade aguda}

O carbofurano utilizado nos experimentos, da marca Sigma-Aldrich, possui grau de pureza de $98 \%$. A solução estoque da substância $\left(100 \mathrm{mg} \mathrm{L}^{-1}\right)$ foi preparada pela diluição do carbofurano em água destilada. Os procedimentos metodológicos foram realizados de acordo com norma padronizada (ABNT, 2004). Os testes de toxicidade aguda consistiram na exposição de 5 neonatas (entre 6 e 24 horas de idade) em 4 réplicas, à diferentes 
concentrações nominais de carbofurano $\left(0,325 ; 0,65 ; 1,3 ; 2,6 ; 5,2\right.$ e $\left.10,4 \mu \mathrm{g} \mathrm{L}^{-1}\right)$. Essa faixa de sensibilidade foi estabelecida baseando-se na $C_{50} 48 \mathrm{~h}$ do cladócero Ceriodaphnia dubia (2,6 $\mathrm{g} \mathrm{L} \mathrm{L}^{-1}$ ) (USEPA, 2005). As soluções das concentrações testadas foram preparadas imediatamente antes dos testes.

Os testes de toxicidade tiveram a duração de 24 e 48 horas e foram realizados em placas de cultivo celular de 6 poços $\left(\operatorname{TPP}^{\circledR}\right)$. Como controle utilizou-se água reconstituída. Foram realizadas quatro repetições tanto para o controle como para cada concentração de carbofurano testada, sendo que em cada réplica foram adicionados $10 \mathrm{~mL}$ de soluçãoteste. Os experimentos foram mantidos na mesma temperatura dos cultivos-estoque, sem iluminação e alimentação. No início dos testes foram realizadas medidas das variáveis $\mathrm{pH}$, condutividade, temperatura e dureza, sendo que todas estas variáveis foram medidas também no final do teste, com exceção da dureza.

\subsection{Tratamento de Dados e Análise Estatística}

Após o período de exposição (24 e 48 horas) procedeu-se à contagem dos organismos imóveis. Se a porcentagem de indivíduos imóveis no controle excedesse $10 \%$, o teste era considerado inválido, o que não ocorreu nesse experimento. Os resultados foram expressos em CE50 - concentração efetiva mediana que causa efeito agudo a $50 \%$ dos organismos no tempo de exposição (ABNT, 2004), com intervalo de confiança de 95\%, utilizando-se o programa estatístico Trimmed Spearmann- Karber (HAMILTON; RUSSO; THURFTON, 1977).

\section{RESULTADOS E DISCUSSÃO}

Durante os testes de toxicidade aguda para a Ceriodaphnia silvestrii, os valores do $\mathrm{pH}$ das soluções testes permaneceram dentro da faixa de 7,1 a 7,6 e não variaram mais do que 0,5 durante 0 teste. A temperatura variou de 24,4 a $25,7{ }^{\circ} \mathrm{C}$, a condutividade elétrica variou de 138,4 a $150,7 \mu \mathrm{S} \mathrm{cm}^{-1}$ e a dureza inicial variou de 40 a $42 \mathrm{mg} \mathrm{CaCO}_{3} \mathrm{~L}^{-}$ 1. Assim, os testes de toxicidade preencheram os critérios de validade estabelecidos nas diretrizes da Associação Brasileira de Normas Técnicas (ABNT, 2005) para C. silvestrii. 
A Figura 2 apresenta o número de indivíduos imóveis de $C$. sivestrii encontrados no controle e nas concentrações testadas de carbofurano durante 24 e 48 horas dois testes de toxicidade aguda realizados. Pela análise da Figura 2, observou-se que, após 24 e 48 horas de exposição ao agrotóxico, 100\% dos organismos de C. silvestrii já apresentaram imobilidade em 5,2 $\mu \mathrm{g} \mathrm{L}^{-1}$ e $2,6 \mu \mathrm{g} \mathrm{L}^{-1}$ de carbofurano, respectivamente. Na Tabela 1 são apresentados os valores da $\mathrm{CE}_{50} 24 \mathrm{~h}$ e $48 \mathrm{~h}$ e os intervalos de confiança obtidos nos dois testes realizados. Os valores médios de $\mathrm{CE}_{50}$ 24-h e $\mathrm{CE}_{50}$ 48-h foram de 1,80 $\mu \mathrm{g} \mathrm{L}^{-1} \mathrm{e}$ $0,75 \mu \mathrm{g} \mathrm{L}^{-1}$, respectivamente.

(a)

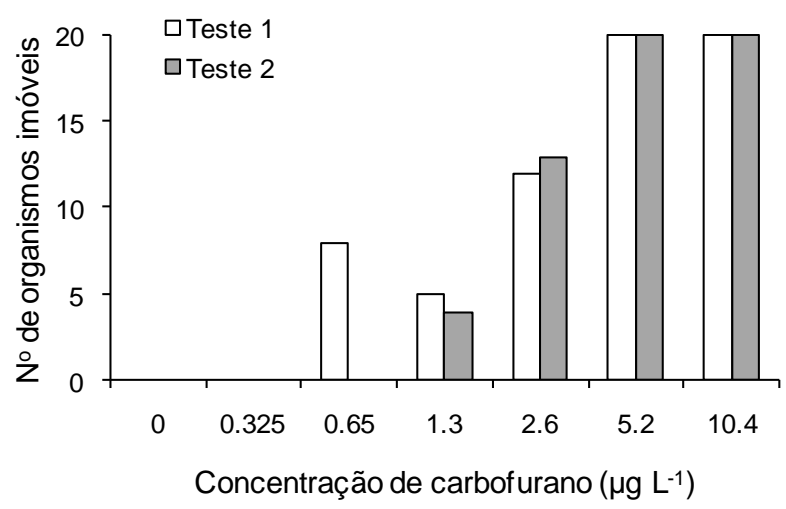

(b)

48 horas

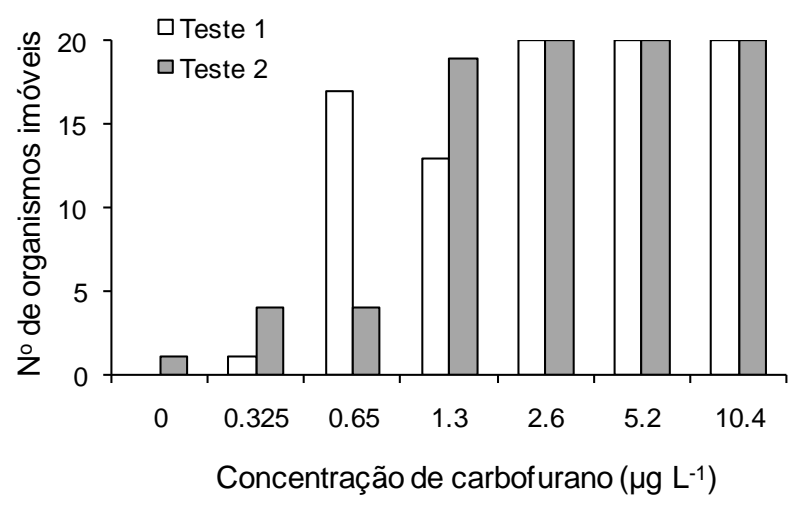

Figura 2: Número de organismos imóveis no controle e nas concentrações testadas para $C$. silvestrii expostos ao agrotóxico carbofurano durante 24 horas (a) e 48 horas (b) nos dois testes de toxicidade aguda.

Nota: O número de organismos imóveis refere-se à somatória das quatro réplicas.

Tabela 1: Valores de $\mathrm{CE}_{50} 24$ e 48 horas do agrotóxico carbofurano e respectivos intervalos de confiança (IC- 95\%) para o cladócero Ceriodaphnia silvestrii em dois testes de toxicidade aguda.

\begin{tabular}{cccc}
\hline Número do teste & Tempo de exposição $(\mathbf{h})$ & $\mathrm{CE}_{50}\left(\mu \mathrm{g} \mathrm{L}^{-1}\right)$ & Intervalo de Confiança (IC-95\%) \\
\hline \multirow{2}{*}{1} & 24 & 1.55 & $1.20-2.00$ \\
& 48 & 0.62 & $0.50-0.76$ \\
2 & 24 & 2.04 & $1.68-2.47$ \\
& 48 & 0.88 & $0.80-0.96$ \\
\hline
\end{tabular}


De acordo com os resultados, observou-se que a espécie nativa Ceriodaphnia silvestrii apresentou alta sensibilidade ao agrotóxico carbofurano, tanto em 24 horas quanto em 48 horas de exposição. Na Tabela 2 são apresentados dados ecotoxicológicos $\left(\mathrm{CE}_{50} / \mathrm{CL}_{50} ; \mu \mathrm{g} \mathrm{\textrm {L } ^ { - 1 } )}\right.$ para diferentes organismos aquáticos expostos ao carbofurano disponíveis na literatura. Ao comparar a $\mathrm{CE}_{50}$ observada para a $C$. silvestrii a de outras espécies aquáticas, percebeu-se que esta foi mais sensível ao carbofurano do que todas as espécies apresentadas da Tabela 2, desde bactérias (Vibrio fischeri) a peixes (Oreochromis niloticus). No entanto, comparações entre espécies devem ser feitas com cautela, pois existem diferentes causas que influenciam na variabilidade na sensibilidade de espécies para um determinado poluente que incluem as características fisiológicas dos animais selecionados (estado de nutrição, sexo, estágio no ciclo de vida), as condições de cultura (temperatura, alimentação, fotoperíodo e dureza da água) e também propriedades das substâncias utilizadas nos ensaios (degradabilidade, solubilidade e pureza) (COWGILL, 1987; RAND, 1995).

Tabela 2: Valores de $\mathrm{CE}_{50} / \mathrm{CL}_{50}\left(\mu \mathrm{g} \mathrm{L}^{-1}\right)$ para diferentes organismos aquáticos expostos ao agrotóxico carbofurano disponíveis na literatura.

\begin{tabular}{|c|c|c|c|}
\hline Organismo-teste & Parâmetro & $\mathrm{CE}_{50} / \mathrm{CL}_{50}\left(\mu \mathrm{g} \mathrm{L}^{-1}\right)$ & Referência \\
\hline Vibrio fischeri & Inibição de luminescência - 30 min & 28900 & Fernández-Alba et al. (2002) \\
\hline Pseudokirchneriella subcapitata & Inibição de crescimento - $72 \mathrm{~h}$ & 2600 & lesce et al. (2006) \\
\hline Chlorella vulgaris & Inibição de crescimento - 96h & 2000 & Ma et al. (2006) \\
\hline Paramecium caudatum & Mortalidade - 3h & 210000 & Hussain et al. (2008) \\
\hline \multirow[t]{2}{*}{ Ceriodaphnia dubia } & Mortalidade - 48h & 2.6 & USEPA (2005) \\
\hline & Mortalidade - 96h & 2.53 & Bailey et al. (1996) \\
\hline Ceriodaphnia silvestrii & Imobilização - 48h & 0.75 & Presente estudo \\
\hline Moina micrura & Mortalidade - 48h & 6.96 & Iwai et al. (2011) \\
\hline Daphnia pulex & Mortalidade - 48h & 35 & Hartman e Martin (1985) \\
\hline \multirow[t]{2}{*}{ Daphnia magna } & Mortalidade - 48h & 18.7 & Dobšíková (2003) \\
\hline & Imobilização - 48h & 59 & Herbrandson et al. (2003) \\
\hline Brachionus calyciflorus & Mortalidade - 24h & 14100 & lesce et al. (2006) \\
\hline Chironomus riparius & Mortalidade - 24h & 27.2 & Ibrahim et al. (1998) \\
\hline Poecilia reticulata & Mortalidade - 96h & 224.5 & Dobšíková (2003) \\
\hline Oreochromis niloticus & Mortalidade - 96h & 220.7 & Pessoa et al. (2011) \\
\hline
\end{tabular}

É importante ressaltar que a espécie nativa $C$. silvestrii foi muito mais sensível do que os cladóceros de regiões temperadas comumente utilizadas em testes 
ecotoxicológicos, como Ceriodaphnia dubia, Daphnia magna e Daphnia pulex (ver Tabela 2). O uso de espécies de clima temperado em regiões tropicais para as avaliações ecotoxicológicas parece ser inadequado, uma vez que as respostas tóxicas de organismos a substâncias químicas são freqüentemente espécie-específicas. Portanto, o uso destas espécies nos trópicos tem sido questionado (DO HONG et al., 2004), uma vez que os seus efeitos podem estar relacionados com variáveis ambientais específicas para cada região.

Com exceção da União Europeia, que estabeleceu um nível máximo de $0,1 \mu \mathrm{g} \mathrm{L}^{-1}$ para cada pesticida (EUROPEAN UNION, 1998), o valor da $C_{50}$ 48-h para C. silvestrii $\left(0,75 \mu \mathrm{g} \mathrm{L}^{-1}\right)$ está abaixo das diretrizes para as concentrações de carbofurano na água potável estabelecidos por vários países, que variam de $5 \mathrm{\mu g} \mathrm{L}^{-1}$ na Austrália a $90 \mu \mathrm{g} \mathrm{L}^{-1}$ no Canadá (WORLD HEALTH ORGANIZATION, 2004), apontando, assim, para um potencial de risco de concentrações de carbofurano legalmente aceitas, pelo menos para alguns organismos aquáticos. No Brasil, a Resolução Conama no 357/05 (BRASIL, 2005), que estabelece padrões de qualidade para corpos d'água e lançamento de efluentes, não contempla o agrotóxico carbofurano. A Portaria 2.914/11 do Ministério da Saúde, que substitui a Portaria 518/04 e estabelece padrões de potabilidade da água para consumo humano, relacionou e limitou o carbofurano em $7 \mu \mathrm{g} \mathrm{L}^{-1}$.

Além dos efeitos diretos causados pelo carbofurano aos organismos, o desaparecimento de herbívoros naturais, como o zooplâncton, devido às concentrações letais de pesticidas, pode indiretamente alterar a abundância e composição da comunidade fitoplanctônica, levando à proliferação de algas (DE LORENZO et al., 2001). Além disso, a falta de alimento para os predadores de zooplâncton, como larvas de peixes, também pode alterar as cadeias alimentares locais.

Assim, observou-se que o uso da espécie nativa $C$. silvestrii em avaliações ecotoxicológicas forneceu dados ecologicamente relevantes sobre a toxicidade do carbofurano que podem dar subsídios para ações futuras voltadas à preservação da diversidade da biota aquática.

\section{CONCLUSÕES}


De acordo com os resultados de toxicidade e comparações com dados da literatura, concluiu-se que a espécie nativa Ceriodaphnia silvestrii apresentou elevada sensibilidade ao agrotóxico carbofurano e foi mais sensível do que as espécies de cladóceros de regiões temperadas. Assim, sugere-se o uso de espécies nativas em avaliações ecotoxicológicas, como a $C$. silvestrii, que podem fornecer mais informações ecologicamente relevantes sobre a toxicidade dos agrotóxicos nos ambientes aquáticos tropicais. No entanto, mais estudos de toxicidade, principalmente de toxicidade crônica e estudos in situ, devem ser realizados para a avaliação dos diferentes efeitos deste composto nos ecossistemas aquáticos, a fim de gerar dados para integrar a avaliação de risco de agrotóxicos e proteger os organismos não-alvo frente à contaminação destes produtos no ambiente.

\section{REFERÊNCIAS}

ABNT - Associação Brasileira de Normas Técnicas NBR 12713. Ecotoxicologia aquática - Toxicidade Aguda - Método de ensaio com Daphnia spp. (Cladocera, Crustacea). Rio de Janeiro, 21p, 2004.

ABNT - Associação Brasileira de Normas Técnicas NBR 13373. Ecotoxicologia aquática - Toxicidade crônica - Método de ensaio com Ceriodaphnia spp. (Crustacea, Cladocera). Rio de Janeiro, 15p, 2005.

BAILEY, H.; DIGIORGIO, C.; KROLL, K.; MILLER, J.; HINTON, D.; STARRETT, G. Development of procedures for identifying pesticide toxicity in ambient waters: carbofuran, diazinon, chlorpyrifos. Environmental Toxicology and Chemistry, v. 15, p.837-845, 1996.

BAILLIEUL, M.; BLUST, R. Analysis of the swimming velocity of cadmium-stressed Daphnia magna. Aquatic Toxicology, v. 44, p.245-254, 1999.

BRASIL. Resolução no 357 de 17 de março de 2005. Dispõe sobre a classificação dos corpos de água e diretrizes ambientais para o seu enquadramento, bem como estabelece as condições e padrões de lançamento de efluentes, e dá outras providências. Brasília, p.30, 2005. 
COLLECTIVE OF AUTHORS SPA (State Phytosanitary Administration): List of the Registered Plant Protection Products. Agrospoj, SPA, 2002.

COWGILL, U.M. Critical analysis of factors affecting the sensitivity of zooplankton and the reproducibility of toxicity test results. Water Research, v. 21, p. 1453-1462, 1987.

DE LORENZO, M.E.; SCOTT, G.I.; ROSS, P.E. Toxicity of pesticides to aquatic microorganisms: A review. Environmental Toxicology and Chemistry, v. 20, p.84-98, 2001.

DOBŠíKOVÁ, R. Acute toxicity of carbofuran to selected species of aquatic and terrestrial organisms. Plant Protection Science, v.39, p.103-108, 2003.

DO HONG, L.C.; BECKER-VAN SLOOTEN, K.; TARRADELLAS, J. Tropical ecotoxicity testing with Ceriodaphnia cornuta. Environmental Toxicology, v. 1, p. 497-504, 2004.

EUROPEAN UNION. Council directive onthe quality of water intended for human consumption. 1998/83/EC of 3 November 1998, annex I. Off J Eur Commun L330:32-54, 1998.

FERNÁNDEZ-ALBA, A.R; GUIL, M.D.H; LÓPEZ, G.D.; CHISTI, Y. Comparative evaluation of the effects of pesticides in acute toxicity luminescence bioassays. Analytica Chimica Acta, v.451, p.195-202, 2002.

FISHER, S.J.; GALINAT, G.F.; BROWN, M.L. Acute toxicity of carbofuran to adult and juvenile flathead chubs. Bulletin of Environmental Contamination and Toxicology, v.63, p. $385-391,1999$.

GRÜTZMACHER, D.D.; GRÜTZMACHER, A.D.; AGOSTINETTO, D.; LOECK, A.E.; ROMAN, R.; PEIXOTO, S.C.; ZANELLA, R. Monitoramento de agrotóxicos em dois mananciais hídricos no sul do Brasil. Revista Brasileira de Engenharia Agrícola e Ambiental, v. 12, p. n.6, p.632-637, 2008.

HAMILTON, M.A.; RUSSO, R.C.; THURFTON, R.B.Trimmed Spearman-Karber method for estimating median lethal concentration in toxicity bioassays. Environmental Science and Technology, v. 11, n. 7, p. 714-719, 1977. 
HARTMAN, W.A.; MARTIN, D.B. Effects of four agricultural pesticides on Daphnia pulex, Lemna minor, and Potamogeton pectinatus. Bulletin of Environmental Contamination and Toxicology, v. 35, p.646-651, 1985.

HERBRANDSON, C.; BRADBURY, S.P; SWACKHAMER, D.L. Influence of solids on acute toxicity of carbofuran to Daphnia magna: I. Interactive effects. Aquatic Toxicology, v.63, p.333-342, 2003.

HUSSAIN, M.M.; AMANCHI, N.R.; SOLANKI, V.R.; BHAGAVATHI., M. Low cost microbioassay test for assessing cytopathological and physiological responses of ciliate model Paramecium caudatum to carbofuran pesticide. Pesticide Biochemistry and Physiology, v. 90, n.1, p.66-70, 2008.

IBRAHIM, H.; KHEIR, R.; HELMI, S.; LEWIS, J.; CRANE, M.. Effects of organophosphorus, carbamate, pyrethroid and organochlorine pesticides, and a heavy metal on survival and cholinesterase activity of Chironomus riparius Meigen. Bulletin of Environmental Contamination and Toxicology, v.60, n.3, p.448-55, 1998.

IESCE, M.R.; DELLA GRECA, M.; CERMOLA, F.; RUBINO, M.; ISIDORI, M.; PASCARELLA, L. 2006. Transformation and ecotoxicity of carbamic pesticides in water. Environmental science and pollution research international, v.13, n.2, p.105-9, 2006.

IWAI, C.B.; SOMPARN, A.; NOLLER, B. Using zooplankton, Moina Micrura Kurz to evaluate the ecotoxicology of Pesticides used in paddy fields of Thailand. Pesticides in the Modern World - Risks and Benefits. Margarita Stoytcheva (ed.), 2011. Disponível em: $<$ http://cdn.intechopen.com/pdfs/21183/InTech-

Using_zooplankton_moina_micrura_kurz_to_evaluate_the_ecotoxicology_of_pesticides_u sed_in_paddy_fields_of_thailand.pdf $>$. Acesso em: 03 set. 2013.

MA, J.; LU, N.; QIN, W.; XU, R.; WANG, Y.; CHEN, X. Differential responses of eight cyanobacterial and green algal species, to carbamate insecticides. Ecotoxicology and Environmental Safety, v.63, n.2, p. 268-74.

MITCHELL, S.E.; HALVES, J; LAMPERT, W. 2004. Coexistence of similar genotypes of Daphnia magna in intermittent populations: response to thermal stress. Oikos, v.106, n.3, p.469-478, 2004. 
MÜLLER, H. Wachstum and phosphatbedarf von Nitzschia actinastroides (Lemn.) v. Goor in statischer und homokontiuierliecher kultur unter phosphatlimitierung. Archiv fuer Hydrobiologie Supplement, v. 38, p. 399-484, 1972.

PESSOA, P.C.; LUCHMANN, K.H.; RIBEIRO, A.B.; VERAS, M.M.; CORREA, J.R.M.B.; NOGUEIRA, A.J.; BAINY, A.C.D.; CARVALHO, P.S.M. Cholinesterase inhibition and behavioral toxicity of carbofuran on Oreochromis niloticus early life stages. Aquatic Toxicology, v.105, p.312-320, 2011.

RAND, G.M. Fundamental Aquatic Toxicology: Effects, Environmental Fate, and Risk Assessment, $2^{\mathrm{a}}$ ed. Taylor \& Francis: USA, cap 31, 1995.

RICHARDS, R.P.; KRAMER, J.W.; BAKER, D.B.; KRIEGER, K.A. Pesticides in rainwater in the northeastern United States. Nature, v.327, p. 129-131, 1987.

SINDAG - Sindicato Nacional da Indústria de Produtos para Defesa Agrícola. Uso de defensivos é intensificado no Brasil. Publicada em 30 de julho de 2012. Disponível em: $<$ http://www.sindag.com.br/noticia.php?News_ID=2278>. Acesso em: 14 ago. 2013.

STEWART, A.J.; KONETSKY, B.K. Longevity and reproduction of Ceriodaphnia dubia in receiving waters. Environmental Toxicology and Chemistry, v.17, p.1165-1171, 1998.

TRAYLER, K.M.; DAVIS, J.A. Sensitivity of Daphnia carinata sensu lato to the insect growth regulator, pyriproxyfen. Ecotoxicology and Environmental Safety, v.33, n.2, p.154-156, 1996.

USEPA - UNITED STATES ENVIRONMENTAL PROTECTION AGENCY. Reregistration Eligibility Science Chapter for Carbofuran - Environmental Fate And Effects Chapter Environmental Risk Assessment And Human Drinking Water Exposure Assessment, 2005.

VALENCIA, E.M.; GUERRERO, J.A.; YUNDA, A.; MARTíNEZ, M.J. Evaluación de la adsorción-desorción de ${ }^{14} \mathrm{C}$-carbofuran y Furadan $3 \mathrm{SC} \circledast$ en tres suelos de Cundinamarca (Colombia). Revista Colombiana de Química, vol.37, n.1, p. 79-91, 2008.

WAITE, D.T.; GROVER, R.; WESCOTT, N.D.; SOMMERSTAD, H.; KARR, L. Pesticides in ground water, surface water and spring runoff in a small Saskatchewan watershed.

Environmental Toxicology and Chemistry, v.11, p.741-748, 1992. 
WORLD HEALTH ORGANIZATION - WHO. Guidelines for Drinking-water Quality. Carbofuran in Drinking-water, 2004. Disponível em:

<http://www.who.int/water_sanitation_health/dwq/chemicals/carbofuran.pdf>. Acesso em: 03 set. 2013. 